\title{
Who takes care of the children? The quantity-quality model revisited*
}

\author{
Michael Lundholm and Henry Ohlsson
}

November 1998

\begin{abstract}
We study the Becker and Lewis (1973) quantity-quality model of children adding an explicit child care time constraint for parents. They can purchase day care or take care of the children themselves. Our results are: (i) If there is a combination of purchased and own care, the effect of income on fertility is ambiguous, even if quantity of children is a normal good in the standard sense. This is the Becker and Lewis (1973) result. (ii) If, however, there only is purchased care, the income effect on fertility is positive when quantity is a normal good. (iii) If, on the other hand, there only is own care, there is a different kind of quantity-quality trade-off. The income effect on fertility is positive if quantity is a closer complement than quality to the consumption of goods.
\end{abstract}

JEL: D1, J13

Keywords: fertility, child care, time constraint, quantity-quality trade-off

\footnotetext{
${ }^{*}$ Correspondence to: Michael Lundholm, Department of Economics, Stockholm University, SE-106 91 Stockholm, Sweden, email <mlu@ne.su.se> or Henry Ohlsson, Department of Economics, Uppsala University, P.O. Box 513, SE-751 20 Uppsala, Sweden, email $<$ henry.ohlsson@nek.uu.se>.

This paper is a part of the project "Wages for women and publicly financed day care" (project F0754/96), financed by the Swedish Council for Research in the Humanities and Social Sciences. We started this work while visiting Institut für Finanzwissenschaft, Humboldt Universität zu Berlin in October 1997. We thank Professor Dr Bengt-Arne Wickström and the University for their hospitality and the financial support to Michael Lundholm. Part of the work was done visiting ERMES, Université Panthéon-Assas, Paris II in May 1998. We thank Professeur Gérard Ballot and Maître de Conférences Ali Skalli for hospitality and financial support to Henry Ohlsson. We have also benefited from comments by seminar participants at Uppsala University.
} 


\section{Introduction}

How does increased family income affect fertility? The standard (tautological) answer is that fertility increases with income if the quantity of children is a normal good. The seminal contribution of Becker and Lewis (1973) (henceforth denoted BL) shows that this answer is seriously misleading. ${ }^{1} \mathrm{~A}$ ceteris paribus increase in quality implies an increase in the marginal rate of substitution between quantity and quality, if quantity is a normal good. But such an increase in quality also increases the relative price of quantity in the BL model unlike standard models where prices are constant. The direction of the change in quantity when income increases is, therefore, indeterminate.

BL use a life time model that includes all phases of life for parents. Sometimes, see Hotz et al. (1997), the perspective of a newly married couple is emphasised. Recent empirical studies, e.g., Connelly (1992), Powell (1997) and Blau and Hagy (1998), adopts this perspective and also recognise that small children require child care, which BL do not. ${ }^{2}$

Our purpose is to study how changes in income affect fertility in the quantity-quality model when parents face an explicit child care time constraint. We assume that the quality of children depends on the type of child care provided and that parents in addition to purchased care (day care) also can take care of the children themselves (own care). ${ }^{3}$ In some cases we replicate the BL results, in other cases we do not.

Our main results are: If there is a combination of purchased and own care, we find that the effect of income on fertility still is ambiguous when the quantity of children is a normal good. Necessary conditions for a solution with both purchased and own care are, however, that the marginal utility of spending time with the children is low and that the marginal utility of an additional child is high. These, in conjunction somewhat odd, ${ }^{4}$ conditions put the attention on the possibility of corner solutions.

If parents purchase all child care (as in the BL model) the ambiguouity of the sign of the income effect on fertility disappears. Fertility is increasing in income if the quantity of children is a normal good in the standard sense.

If parents take care of the children themselves, a different quantity-quality

\footnotetext{
${ }^{1}$ For an early discussion see Becker (1960) and for further development Becker and Tomes (1976). For policy discussions see, e.g., Batina (1986), Cigno (1983, 1986), Nerlove et al. $(1984,1986)$.

${ }^{2}$ See also Lundholm and Ohlsson (1998) who apply the same perspective to analyse wage determination and female labour force participation.

${ }^{3}$ We also assume that parents without constraints can choose how much day care parents they want to purchase.

${ }^{4}$ Odd since parents on the margin like having more children but dislike spending more time with them.
} 
trade-off emerges. The income effect on fertility is positive if the quantity of children is a closer complement to consumption goods than the quality of children.

In section 2 we describe our generalisation of the BL model. Section 3 derives the results and section 4 concludes the note.

\section{Model}

A parent reproduces asexually and chooses the quantity of children $n \in N=$ $\left\{n \in \mathbb{R}_{+}: n \geq 1\right\}$. Child quality $q \in \mathbb{R}_{+}$is only acquired through child care, which is purchased by the parent or produced by herself. Purchased quality per child equals the number of purchased hours of day care $d \in \mathbb{R}_{+}$ during which the child gets the full attention of a care taker. Producing the care herself, the parent spends $c \in \mathbb{R}_{+}$of her own time to take care of her $n$ children. The total care time per child is $d+c$. This must not be less than the total time $D$ during which each child needs care; $D \cdot d+c$.

Average quality of own care for each child equals the time during which a child gets the full attention of the care taker; i.e., $\frac{c}{n}$. Purchased and own quality are perfect substitutes and the parent treats all children identically so that average quality is $q=d+\frac{c}{n}$ if $d+\frac{c}{n} \cdot D$, else $q=D$. Hence, $q=D-\frac{(n-1)}{n} c$ and $\partial q / \partial c=n^{-1}-1 \cdot 0$. Substitution of day care for own care reduces average quality when the child care time constraint is binding.

Working hours $\bar{h} \in \mathbb{R}_{++}$are fixed and paid the wage rate $w$. Income is spent on own consumption $\left(x \in \mathbb{R}_{+}\right)$, the price of which is numeraire and normalised to unity, or on purchased quality $p n d$, where $p$ is the unit price of purchased day care, $n$ is the number of children, and $d$ is the quantity of purchased care. The parent's budget constraint is $w \bar{h}=x+p n d$.

Total time $T$ (naturally also $D \cdot T$ ) is spent on market work, taking care of one's children and leisure time $\left(\ell \in \mathbb{R}_{+}\right): T-\bar{h}=c+\ell$.

Parents have preferences represented by the quasi-concave utility function $U^{*}: \mathbb{R}_{+}^{4} \times \mathbb{R}_{++} \times N$ defined by $U^{*}(x, \bar{h}, c, \ell, q, n)$. We use the notation $U_{1}^{*}=\frac{\partial U^{*}(\cdot)}{\partial x}$ etc to denote the partial derivatives and assume that $U_{1}^{*}>0$, $U_{2}^{*}<0, U_{3}^{*} \gtreqless 0, U_{4}^{*}>0, U_{5}^{*}>0$ and $U_{6}^{*}>0$. Hence, we do not make any particular assumption about how the parent values the time spent with her own children.

Combining the time constraints yields $T-D+d \geq \bar{h}+\ell$, but $U_{4}^{*}>0$ implies that leisure can be increased to increase utility so that all available time is used and the inequality disappears. Then $c+d=D$ implies that $0 \cdot d \cdot D$; i.e., $d>D$ is never optimal. With $U_{1}^{*}>0$ a similar argument can be made for the budget constraint. 
We assume that parents have more than one child so that the child care time constraint is invoked. The problem of a parent can be written as

$$
\max _{x, d, n} U(x, d, n) \text { s.t. } w \bar{h}=x+\text { pnd and } 0 \cdot d \cdot D,
$$

where $U(x, d, n)=U^{*}\left(x, \bar{h}, D-d, T-\bar{h}-D+d, d+\frac{D-d}{n}, n\right)$. This problem has the following first order conditions for $x^{*}>0$ and $n^{*}>1$

$$
\begin{array}{rl}
U_{x}-\lambda^{*} & =0 \\
U_{d}-\lambda^{*} p n^{*}-\mu^{*} & 0 \quad d^{*} \geq 0, \\
U_{n}-\lambda^{*} p d^{*} & =0 \\
w \bar{h}-x^{*}-p n^{*} d^{*} & =0 \text { and } \\
D-d^{*} & \geq 0 \quad \mu^{*} \geq 0,
\end{array}
$$

where $\lambda>0$ and $\mu$ are the Lagrange multipliers associated with the constraints.

\section{Quality vs. quantity}

We now revisit the problem of quantity versus quality of children and ask how fertility is affected by income changes in this new setup. We make the analysis in three steps: We consider subsequently (1) the interior solution when child care is arranged through a combination of purchased care and own care, (2) the corner solution where the parent purchases all child care and (3) the corner solution where the parent produces all child care herself.

\subsection{Purchased and own care: $d^{*} \in(0, D)$}

In an interior solution the parent is using purchased as well as own care. Then (2b) is strictly binding and $\mu^{*}=0$ so that $d^{*} \in(0, D)$. Let the (assumed) unique solution satisfying these first order conditions $(2 \mathrm{a})-(2 \mathrm{c})$ be denoted $(x(\bar{y}), q(\bar{y}), n(\bar{y}))$, where $\bar{y}=w \bar{h}$. The main issue is how fertility is affected by income changes; i.e., what is the sign of $\frac{\partial n}{\partial \bar{y}}$ ? Consider now the optimal non-linear solution evaluated in a linear model. In such a model we can write the budget as $I=p_{n} \tilde{n}+p_{q} \tilde{q}+x$, where $I=\bar{y}+p q(\bar{y}) n(\bar{y})$ is full income, $p_{n}=p q(\bar{y})$ and $p_{q}=p n(\bar{y})$. Let $\tilde{S}_{i j}$ denote the substitution effect in the 
linear model where $i, j=p, q$. Standard symmetry gives $\tilde{S}_{i j}=\tilde{S}_{j i}$. Then we have

$$
\frac{\partial n}{\partial \bar{y}}=\frac{\frac{\partial \tilde{n}(I)}{\partial I}\left(p \tilde{S}_{q n}-1\right)-\frac{\partial \tilde{q}(I)}{\partial I} p \tilde{S}_{n n}}{p^{2} \tilde{S}_{q q} \tilde{S}_{n n}-\left(p \tilde{S}_{q n}-1\right)^{2}}
$$

where $\frac{\partial \tilde{n}(I)}{\partial I}$ and $\frac{\partial \tilde{q}(I)}{\partial I}$ are standard income effects. Normality of the quantity of children in the standard sense implies $\frac{\partial \tilde{n}(I)}{\partial I}>0$, but this is not sufficient to sign equation (3); see Razin and Sadka (1995, p.20f) for a discussion about various conditions signing (3).

Necessary conditions for an interior solution are that the marginal utilities of purchased care and quantity are positive; i.e., $U_{d}>0$ and $U_{n}>0$. Although these marginal utilities look similar to the marginal utilities in BL we see the difference clearly if we express the derivatives of $U$ in terms of derivatives of $U^{*}$ :

$$
\begin{aligned}
& U_{d}=-U_{3}^{*}+U_{4}^{*}+\frac{n-1}{n} U_{5}^{*} \gtreqless 0 \text { and } \\
& U_{n}=-\frac{D-d}{n^{2}} U_{5}^{*}+U_{6}^{*} \gtreqless 0 .
\end{aligned}
$$

Utility is affected through three different channels when the parent purchases an additional unit of day care. First, by the child care time constraint, the amount of time spent with children is reduced. This reduces utility if the parent likes to be with the children. Second, by the time constraint, more leisure time becomes available since working hours are fixed, which increases utility. Third, the quality per child is affected. As a direct effect, quality increases when the purchased care is increased. But since own care is reduced, there is also a counteracting indirect effect that reduces quality. However, when the parent purchases one more unit of care per child, the quality is increased by one unit while the reduction of own care with one unit only reduces quality with $n^{-1}$ units. Therefore, the quality effect is positive since we assume that $n^{*}>1$. This means that the marginal utility of purchased care is positive if the second and third effects dominate the first effect.

When the quantity of children is increased marginally there will be a direct positive effect on utility and an indirect negative effect through reduced quality. If the direct effect dominates the indirect effect, then additional children will increase utility.

We can note that the necessary condition is consistent with a parent disliking spending own time to take care of the children; $U_{3}^{*}<0$. Sufficient 
conditions for $U_{d}>0$ and $U_{n}>0$, however, are that $U_{3}^{*}<0$ and $d^{*}=D$. But these conditions are inconsistent with an interior solution and will move us to the corner solution where all child care is purchased.

\subsection{Only purchased care: $d^{*}=D$}

We start by noting that when all day care is purchased then $d^{*}=D$ is optimal; i.e., the parent never prefers to increase $d$ above $D$ so $\mu^{*}=0$. This implies that total quality is exogenously given by $q=D$. The problem of quantity and quality interaction now disappears since quality is, in a sense, exogenously given when we consider the effect of income changes.

Under this restriction and with elimination of the budget constraint the solution is characterised by (2a) and (2c). The second order condition, $\Delta:=$ $p^{2} U_{x x}+2 p U_{x n}+U_{n n}<0$, is satisfied since $U^{*}$ is quasi-concave and $U_{x x}=U_{11}^{*}$, $U_{x n}=U_{16}^{*}$, and $U_{n n}=U_{66}^{*}$. Differentiating the first order condition with respect to the quantity of children $n$ and exogenous income $\bar{y}:=w \bar{h}$ gives the income effect,

$$
\frac{\partial n}{\partial \bar{y}}=\frac{1}{\Delta}\left(p U_{x x}-U_{x n}\right)
$$

which is positive if the quantity of children is a normal good (i.e., $p U_{x x}-$ $U_{x n}<0$ ) and negative if the quantity of children is an inferior good (i.e., $\left.p U_{x x}-U_{x n}>0\right)$, in the standard sense. Suppose that $U^{*}$ is not only quasiconcave but also concave so that $U_{x x}<0$. Then the complementarity of consumption of goods and the quantity of children (in the sense of $U_{x n}>0$ ) is a sufficient condition for the quantity of children to be a normal good. ${ }^{5}$ Note that in this case the general non-linear model coincides with the linear approximation everywhere and not only in the optimal allocation of the nonlinear model; i.e., $\frac{\partial n}{\partial y}=\frac{\partial \tilde{n}}{\partial I}$.

Even if the the price of quality, in the sense of BL, is changed when the number of children is changed there will be no secondary effect on quality since quality is set by the child care time constraint. We conclude that using the BL assumption of no own care together with the child care time constraint eliminates the interaction between quality and quantity. In the next section we allow for the possibility of own care. We show that this can reintroduce a quantity-quality trade-off, even in a corner solution.

\footnotetext{
${ }^{5}$ Note that this definition of complementarity, the Pareto-Georgescu criterion, may deviate from the standard definition that the compensated cross elasticity should be positive. See e.g., Samuelson (1974).
} 


\subsection{Only own care: $d^{*}=0$}

Suppose that there is only own care; i.e., $d^{*}=0$. Then $(2 \mathrm{~b})$ implies $U_{d}-$ $\lambda^{*} p n^{*} \cdot 0$, possibly with a strict inequality. This situation may occur when the parent loves staying home to take care of the children and, therefore, $U_{3}^{*}>0$ and also relatively high. Consumption is given by equation (2d). When we consider the effect on an income increase the first order condition describing individual behaviour simplifies to $U_{n}=0$, with the second order condition $\Delta_{2}:=U_{n n}<0$. In the notation of the general model we have

$$
\begin{array}{r}
U_{n}=-\frac{D}{n^{2}} U_{5}^{*}+U_{6}^{*}=0 \quad \text { and } \\
U_{n n}=\frac{D}{n^{3}} U_{5}^{*}+\frac{D^{2}}{n^{4}} U_{55}^{*}-\frac{2 D}{n^{2}} U_{56}^{*}+U_{66}^{*}<0 .
\end{array}
$$

Note that if we assume that $U_{n}>0$ we would exclude the case of only own care given the general model. Note also that if $U_{n n}<0$ then we are safe and can continue our analysis with the comparative static analysis. However, this is not necessarily the case. If the marginal utility of quality is high when the number of children is low or if quantity and quality are substitutes, then one possibility is that the number of children (which we have assumed is larger than one) that satisfy the first order condition is a (local) minimum and also that the (global) maximum is the corner solution $n=1$. There is also a possibility that there are multiple solutions, all implying the same utility, satisfying the first order condition. In the following we disregard from these problems and assume that the second order condition is satisfied so that there exists a unique interior solution for the quantity of children.

Total differentiation with respect exogenous income $\bar{y}$ and the quantity of children then gives us

$$
\left.\frac{\partial n}{\partial \bar{y}}=\frac{1}{\Delta_{2}} \frac{D}{n^{2}} U_{51}^{*}-U_{61}^{*}\right],
$$

where the denominator is negative by the second order condition. The equation (7) shows the quantity-quality trade-off when there is no purchased care. This condition states that if the quantity of children is a closer complement to the consumption of goods than quality in the sense of $\frac{D}{n^{2}} U_{51}^{*}-U_{61}^{*}>0$, then increased exogenous income will increase the quantity of children. This would be the case, for example if quality is a substitute and quantity is a complement to the consumption of goods. Since $\frac{\partial q}{\partial n}=-n^{-2}<0$ the quality of children will be reduced. However, if the quality of children is a 
closer complement to consumption of goods than quantity in the sense of $\frac{D}{n^{2}} U_{51}^{*}-U_{61}^{*}<0$, then increased exogenous income will reduce the quantity of children and also increase the quality of children.

\section{Conclusions}

Becker and Lewis (1973) show that the effect of income on fertility is ambiguous, even if the quantity of children is a normal good in the standard sense. In this paper we have shown that this result extends to a situation where parents face an explicit child care time constraint and choose a combination of purchased day care and child care produced by themselves.

However, if only purchased day care is used, the average quality is determined by the child care time constraint and the quantity-quality trade-off disappears. The effect of income on fertility now is positive if the quantity of children is a normal good in the standard sense.

On the other hand, if parents care for the children themselves, a different kind of quantity-quality trade-off arises. More children reduce the quality of an hour of the parent's time spent on child care. The income effect of fertility now is positive if the quantity of children is a closer complement than quality to the consumption of goods. 


\section{References}

R. G. Batina. The optimal linear income tax with credits contingent on fertility. Journal of Public Economics, 30(2):219-235, July 1986.

G. S. Becker. An economic analysis of fertility. In Demographic and Economic Change in Developed Countries, Universities-National Bureau Conference Series 11. Princeton University Press, Princeton, NJ, 1960.

G. S. Becker and H. G. Lewis. On the interaction between the quantity and quality of children. Journal of Political Economy, 81(2, Part II):S279-S288, March-April 1973.

G. S. Becker and N. Tomes. Child endowments and the quantity and quality of children. Journal of Political Economics, 84(4, Part II):S143-S162, August 1976.

D. H. Blau and A. P. Hagy. The demand for quality in child care. Journal of Political Economy, 106(1):104-146, February 1998.

A. Cigno. On optimal family allowances. Oxford Economic Papers, 35(1): 13-22, March 1983.

A. Cigno. Fertility and the tax-benefit system: A reconsideration of the theory of family taxation. Economic Journal, 96(384):1035-1051, December 1986.

R. Connelly. The effect of child care costs on married women's labor force participation. Review of Economics and Statistics, 74(1):83-90, February 1992.

V. J. Hotz, J. A. Klerman, and R. J. Willis. The economics of fertility in developed countries: A survey. In M. R. Rosenzweig and O. Stark, editors, Handbook of Population and Family Economics, Volume 1A, chapter 7, pages 275-347. North-Holland, Amsterdam, 1997.

M. Lundholm and H. Ohlsson. Wages taxes and publicly provided day care. Journal of Population Economics, 11(2):185-204, May 1998.

M. Nerlove, A. Razin, and E. Sadka. Income distribution policies with endogenous fertility. Journal of Public Economics, 24(2):221-230, July 1984. 
M. Nerlove, A. Razin, and E. Sadka. Some welfare theoretic implications of endogenous fertility. International Economic Review, 27(1):3-31, February 1986.

L. M. Powell. The impact of child care costs on the labour supply of married mother: Evidence from Canada. Canadian Journal of Economics, 30(3): 577-594, August 1997.

A. Razin and E. Sadka. Population Economics. MIT Press, Cambridge, MA and London, England, 1995.

P. Samuelson. Complementarity. An essay on the 40th anniversary of the Hicks-Allen revolution in demand theory. Journal of Economic Literature, 12(4):1255-1289, December 1974. 